\title{
Effect of Silane Coupling Agent Chemistry on Electrical Breakdown across Hybrid Organic- Inorganic Insulating Films
}

\section{Citation}

Diebold, Roger Mitchell, Michael J. Gordon, and David R. Clarke. 2014. "Effect of Silane Coupling Agent Chemistry on Electrical Breakdown across Hybrid Organic-Inorganic Insulating Films." ACS Applied Materials and Interfaces 6 (15) (July 22): 11932-11939. doi:10.1021/am504305k. http://dx.doi.org/10.1021/am504305k.

\section{Published Version}

doi:10.1021/am504305k

\section{Permanent link}

http://nrs.harvard.edu/urn-3:HUL.InstRepos:12718791

\section{Terms of Use}

This article was downloaded from Harvard University's DASH repository, and is made available under the terms and conditions applicable to Other Posted Material, as set forth at http:// nrs.harvard.edu/urn-3:HUL.InstRepos:dash.current.terms-of-use\#LAA

\section{Share Your Story}

The Harvard community has made this article openly available.

Please share how this access benefits you. Submit a story.

Accessibility 


\title{
Effect of silane coupling agent chemistry on
}

\section{electrical breakdown across hybrid organic-inorganic}

\section{insulating films}

Roger M. Diebold, ${ }^{1}$ Michael J. Gordon, ${ }^{2}$ David R. Clarke, ${ }^{1}$

${ }^{1}$ School of Engineering and Applied Sciences, Harvard University, Cambridge, Massachusetts, 02138, USA

${ }^{2}$ Department of Chemical Engineering, University of California, Santa Barbara, Santa Barbara, California, 93106-5080

KEYWORDS: Self assembled monolayer, dielectric breakdown, silane coupling agents, interfacial, electrical insulators, Weibull analysis, conductive atomic force microscopy

\begin{abstract}
Dielectric breakdown measurements were conducted on self-assembled monolayer (SAM)/native silicon oxide hybrid dielectrics using conductive atomic force microscopy (C-AFM). By depositing silane coupling agents (SCAs) through a diffusional barrier layer, SAM roughness was decoupled from chemistry to compare the chemical effects of exposed R-group functionality on
\end{abstract}

- Author to whom correspondence should be addressed. Electronic mail: rdiebold@seas.harvard.edu. 
dielectric breakdown. Using Weibull and current-voltage (I-V) analysis, the breakdown strength was observed to be independent of SCA R-group length, and the addition of a SAM was seen to improve the breakdown strength relative to native silicon oxide by up to $158 \%$. Fluorinated SCAs were observed to suppress tunneling leakage and exhibited increased breakdown strength relative to their hydrocarbon analogs. Electron trapping, scattering, or attachment processes inherent to the fluorinated moieties are thought to be the origin of the improved breakdown properties.

\section{INTRODUCTION}

In the concerted effort to increase transistor density in integrated circuits, self-assembled monolayers (SAMs) have been identified as promising materials for transistor gate insulators. When coupled with inorganic layers, SAMs demonstrate exceptionally low leakage currents, ${ }^{1-2}$ while contributing $<2 \mathrm{~nm}$ to the gate insulator thickness, permitting high capacitance without the leakage currents traditionally associated with thin $(<2 \mathrm{~nm})$ silicon dioxide insulators. In addition to reducing the gate insulator thickness, low leakage increases on/off ratios, lowers power consumption, and increases the permissible operating gate bias of transistors. As such, SAMs have been used with oxides as insulators for functional devices ${ }^{3-5}$ and probing charge transfer mechanisms. ${ }^{6-8}$ However, the SAMs used are often complex and multilayer in nature, illustrating the need for a more fundamental understanding of how specific chemical groups within the SAM act as electrical insulators to facilitate selection of appropriate compounds. In particular, elucidating the relationship between SAM chemistry and dielectric breakdown 
is necessary to fabricate reliable hybrid gate insulators. In this article, we present through-thickness (strike) breakdown data for a variety of silane coupling agent (SCA) SAMs on native silicon oxide as evaluated by conductive atomic force microscopy (CAFM). A barrier layer deposition technique ${ }^{9}$ is employed to deposit reactive SCAs as SAMs to achieve comparable sample morphologies across a wide variety of chemistries. Weibull and current-voltage (I-V) analysis are used to interpret these data to correlate breakdown metrics and mechanisms with SAM chemistry (i.e., SCA length, halogenation, and number of binding groups).

Measuring dielectric breakdown is challenging because it is highly sensitive to electric field distributions within the material. ${ }^{10}$ For example, high electric fields can be found close to filler particles or defects in a matrix because of dielectric constant mismatch as found in nanocomposites. ${ }^{11-12}$ As smaller insulator volumes are probed, fewer defects are encountered, which tends to increase the breakdown strength towards an ultimate intrinsic limit. ${ }^{13}$ C-AFM was originally used in the 1990 's as a technique to investigate electrical breakdown of nanoscale materials, ${ }^{14-15}$ with significant effort concentrated on evaluating the breakdown of silica and hafnia inorganic insulating layers for transistor gate applications. ${ }^{16-21}$ More recently, C-AFM breakdown measurements have been performed on nanoscale organic insulator systems. ${ }^{22-23}$ For several reasons, we believe that C-AFM is an effective method for evaluating dielectric breakdown on nanoscale thin films: multiple I-V curves can be collected with relative ease through automated routines, the high lateral resolution allows avoidance of obvious defects, and the choice of a solid doped diamond tip, in combination with a current limiting resistor, affords a relatively 
reliable electrode geometry. Additionally, if a constant, small contact force $(<10 \mathrm{nN})$ is applied by the tip to the SAM, the organic molecules have been shown not to appreciably deform. $^{22,24-25}$ Other methods of conducting breakdown investigations on SAM-coated surfaces can involve metal evaporation through a shadow mask, which may more closely approximate practical device fabrication conditions than C-AFM, the technique can lead to diffusion of metal atoms into the $\mathrm{SAM},{ }^{26}$ thereby increasing the probability that defects are measured, rather than intrinsic electrical properties. Although thiol-terminated SAMs reduce the incidence of gold penetration, this is a special case, and the use of other nonthiolated SCA molecules is often desired. However, we believe the distinction between local breakdown observed by C-AFM to provide complementary information relative to sampling weakest link statistics obtained via device testing. In this investigation, we make use of the fine spatial resolution afforded by C-AFM to minimize exposure to film defects, electrically interrogate SAMs on oxide in a highly local manner, and further understand the relationship between chemical and electrical breakdown characteristics of such systems.

In addition to the use of C-AFM to measure dielectric breakdown of nanoscale insulator films, it is imperative that the sample surface is homogeneous to compare results between multiple organic monolayer chemistries, especially when evaluating those prone to forming agglomerates. Through creating a viscous oil barrier on the sample surface before SCA vapor deposition, agglomerates can be screened out through differences in diffusion rates with individual molecules. Relative to SCA deposition without the barrier layer, this method ${ }^{9}$ allows smoother monolayers to be formed under ambient conditions 
and facilitates the formation of similar monolayer morphologies and densities somewhat independent of SCA reactivity. For example, inherent differences in SCA reactivity and consequent likelihood to bond to the surface, such as between trichlorosilanes and monochlorosilanes, influence their ability to pack on the surface. If left to form the densest monolayer possible for a particular SCA, breakdown measurements may result in probing packing density as a variable, observed to affect breakdown strength, ${ }^{27}$ rather than chemistry. Intentional aggregate removal and disordered monolayer formation through the barrier layer deposition technique is seen as a way forward in isolating the effect of chemistry on dielectric breakdown.

\section{EXPERIMENTAL DETAILS}

A. Wafer preparation:

A single degenerately doped silicon wafer (Sb-doped, (111), $5 \times 10^{-3}-1.8 \times 10^{-3} \Omega$-cm, prime grade, $0.5 \mathrm{~mm}$ thick, University Wafer, Inc.) was diced into $1 \mathrm{~cm}$ squares and sonicated in acetone for 5 mins before rinsing in isopropanol and deionized (DI) water in a cleanroom environment. After blow drying with nitrogen, the wafer pieces were immersed in hot $\left(90^{\circ} \mathrm{C}\right)$ Nanostrip (Cyantek, Co.) for 1 min to remove any remaining organics, rinsed with DI water, immersed in hydrofluoric acid (J.T. Baker, Co., 49\%), rinsed with DI water, and immersed in hot Nanostrip for 6 hrs to grow a native oxide. After rinsing in DI water, the samples were dehydrated $\left(115^{\circ} \mathrm{C}, 15\right.$ mins $)$ prior to measuring the native oxide thickness by spectroscopic ellipsometry, yielding an average thickness of $1.5 \mathrm{~nm}$. To give an indication of the repeatability of the oxide growth, ten different regions of a single, cleaned and oxidized $100 \mathrm{~mm}$ diameter wafer were 
measured via ellipsometry, whose standard deviation was $<0.05 \mathrm{~nm}$. To prevent subsequent surface contamination, the samples were vacuum sealed under dry argon until tested.

\section{B. SCA deposition:}

All SCAs used were purchased from Gelest, Inc. and filtered through a $0.2 \mu \mathrm{m}$ PTFE syringe filter immediately before deposition; a complete list of chemical names and

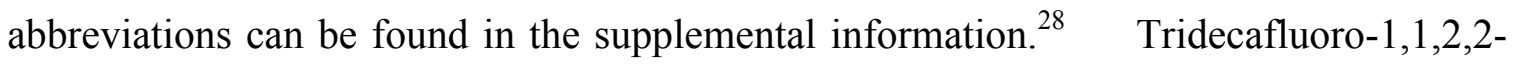
tetrahydrooctyldimethylmethoxysilane (tDF-dMeM) was not commercially available and was produced from its chlorosilane analog ${ }^{29}$ through the addition of anhydrous methanol under an inert atmosphere.

The experimental procedure for forming SCA SAMs on silicon dioxide has been published previously, ${ }^{9}$ and is outlined briefly as follows. A $1 \% \mathrm{v} / \mathrm{v}$ solution of silicone oil (Dow Corning Fluid 200, 50cSt) in heptane (Sigma Aldrich) was spin coated on a wafer (5 krpm, $40 \mathrm{~s}$ ) to form a $\sim 70 \mathrm{~nm}$ barrier layer. SCA molecules were vapor deposited onto the samples in a $1 \mathrm{~L}$ Teflon jar for $1 \mathrm{hr}$ in a dry argon atmosphere. The silicone oil was removed through sonication in xylenes (Sigma Aldrich) for $1 \mathrm{hr}$ and acetone for 30 mins, then rinsed with isopropanol and DI water; the samples were subsequently heat treated $\left(115^{\circ} \mathrm{C}, 10 \mathrm{mins}\right)$ to promote $\mathrm{SCA}$ fixation to the surface via covalent bonds. Two controls were also exposed to the same cleaning, washing, and heating regimen: one without silicone oil or SCA, and one with only silicone oil. SCA chemical names, abbreviations, and structures are found in Table I. 


Silane coupling agent (SCA)
octyldimethylmethoxysilane

Table I. Chemical structures of SCAs used in this investigation and their abbreviations for the purposes of this manuscript. Methoxy binding groups are highlighted in red, and chloro groups are highlighted in green.

\section{Film thickness determination:}

Spectroscopic ellipsometry (WVASE32, J.A. Woollam, Inc.) was conducted over $\lambda=$ $500-1000 \mathrm{~nm}$ at $55^{\circ}, 65^{\circ}$, and $75^{\circ}$ relative to the sample normal to determine SAM thicknesses. The thicknesses were fit using a $1.5 \mathrm{~nm}$ (rounded) fixed oxide layer below a Cauchy layer (initial guess $2 \mathrm{~nm}$ ) with refractive indices corresponding to the SCA bulk values, using thermal $\mathrm{SiO}_{2}$ and $\mathrm{Si}$ layer optical constants provided by J.A. Woollam, Co. ${ }^{30}$ and Cauchy parameters as follows: $A_{n}=($ SCA bulk refractive index $), B_{n}=0.01, C_{n}$ $=0, \mathrm{k}$ amplitude $=0$, exponent $=1.5$, band edge $=400 \mathrm{~nm}$. Although silicone oil is an 
adequate barrier material for a wide range of SCA chemistries, the fluorinated hydrocarbon trichlorosilanes (nF-tC, $\mathrm{tDF}-\mathrm{tC}$, and $\mathrm{hDF}-\mathrm{tC})$ yielded more aggregates than desirable because of the highly reactive trichlorosilane moieties, in combination with the use of a relatively thin barrier layer. Consequently, the trichlorosilane SAM thicknesses were greater than expected as measured by ellipsometry, whose data were averaged over a $1.5 \mathrm{~mm}$ diameter spot size. Nevertheless, agglomerates, which skewed trichlorofunctional SAM thickness values, were easily observed during the C-AFM tests and could be avoided; it was assumed that the thickness values from another set of identically produced yet agglomerate-free samples would suffice to calculate breakdown strengths for the trichlorosilanes. The difficulty in reproducing these fluorinated SCA SAMs has since been improved with the use of PFPE barrier layers. ${ }^{9}$ It must be emphasized that the conclusions of this article are not affected by this thickness assumption; a direct comparison between fluorinated and non-fluorinated SCA analogs was made, both of which repeatedly formed smooth SAMs. The trichlorosilane data were included to lend additional support to the conclusions as the local SAM thicknesses are unaffected by the presence of agglomerates.

\section{C-AFM breakdown measurements:}

After SCA deposition, samples were tested with boron-doped solid diamond C-AFM tips of nominal radius $\sim 35 \mathrm{~nm}$ (NaDiaProbes, ND-CTIR1-4, $0.04 \mathrm{~N} / \mathrm{m}$, Nanoscience Instruments, Inc.) in conjunction with an Asylum MFP-3D AFM. A Keithley 2400 sourcemeter was electrically connected directly to the cantilever holder (positive lead) with a $1 \mathrm{G} \Omega$ current limiting resistor in series, and ohmic connection was made with the 
doped Si substrate (negative lead) through an indium solder contact. The sample was first scanned to clean the tip $(7 \mathrm{nN}, 1 \mathrm{~Hz})$ and image the sample surface $(256 \times 256$ lines, $40 \mu \mathrm{m} \times 40 \mu \mathrm{m}$ image). The image was then used to select the flattest areas to test, as demonstrated in Figure 1. The tip was then moved to a clean region of the sample and a $7 \mathrm{nN}$ contact force was exerted by the tip on the surface, low enough to prevent significant SAM deformation ${ }^{25}$ but high enough to ensure an ohmic contact on a clean platinum surface, which was verified before each sample test. The spring constant of the AFM cantilever was determined using a force-deflection curve in conjunction with the thermal noise method, which employs the equipartition theorem. ${ }^{31}$ Twenty five I-V measurements were made for each sample in a $5 \times 5$ square array, stepping $5 \mu \mathrm{m}$ between tests, during which a ramped bias of $0.75 \mathrm{~V} / \mathrm{s}$ was applied. When the breakdown threshold of $5 \mathrm{nA}$ was detected, the measurement was terminated through the use of a LabVIEW control program. This current level was chosen as a breakdown criterion as it is large relative to the pA level leakage observed at low bias, but also low enough to preserve integrity of the C-AFM tip over multiple tests. The first breakdown value for each sample was discarded as it was found in every case to be unusually large, likely caused by the accumulation of adsorbed molecules and naturally occurring carbon on the tip surface during the initial scan. After each sample was tested in breakdown, the tip's contact resistance was checked on an evaporated platinum film on silicon. Hard scanning (46 $\mathrm{nN}$ applied contact force) and heating (application of current) allowed removal or desorption of organic contaminants until ohmic contact was made consistently to the platinum surface using a $7 \mathrm{nN}$ applied contact force. 


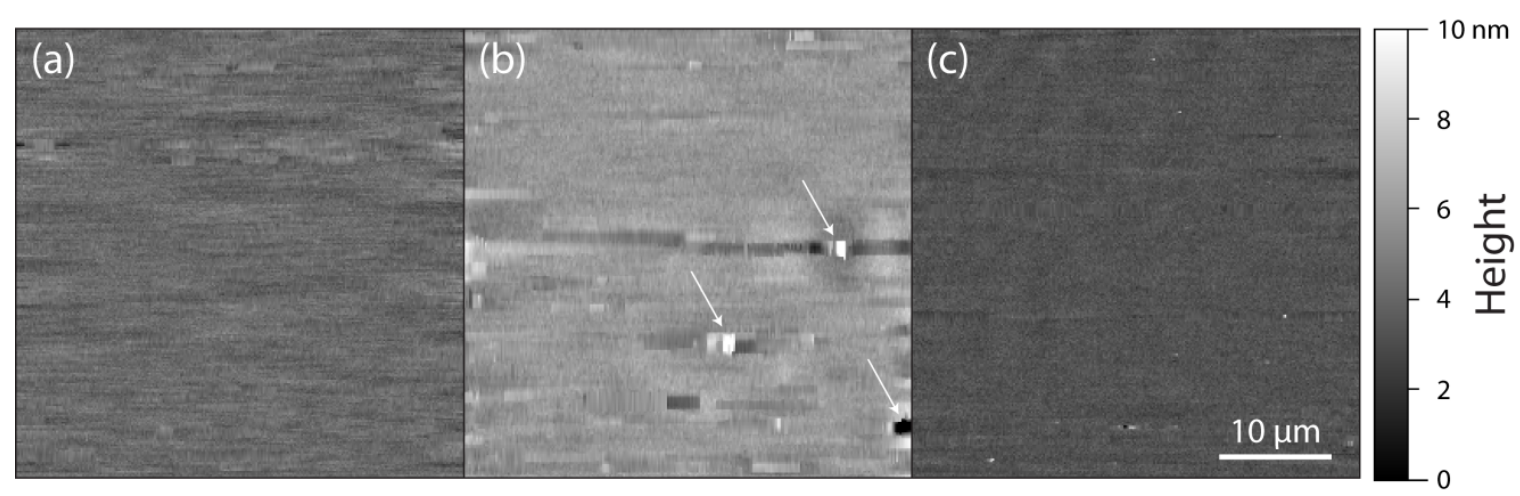

Figure 1. Contact mode AFM topographic images of samples taken prior to breakdown tests. (a) native silicon oxide without silicone oil, (b) tDF-dMeM with silicone oil, and (c) o-dMeM with silicone oil. All scans are $40 \mu \mathrm{m} \times 40 \mu \mathrm{m}$. Agglomerates seen in (b) (arrows) were easily avoided during I-V testing.

Figure 1 shows morphology typical of the SCA SAM samples, and Table II summarizes roughness measurements of the SAM films. The few agglomerates encountered were avoided during the test as only a $25 \mu \mathrm{m} \times 25 \mu \mathrm{m}$ agglomerate-free region was necessary. Image quality was compared before and after testing to ensure that the tip geometry had not been altered. 


\begin{tabular}{|c|c|c|c|}
\hline SCA & $\begin{array}{c}\text { RMS } \\
\text { roughness: } \\
40 \mu \mathrm{m} x \\
40 \mu \mathrm{m} \text { scan } \\
(\mathrm{nm})\end{array}$ & $\begin{array}{c}\text { RMS } \\
\text { roughness: } \\
25 \mu \mathrm{m} x \\
25 \mu \mathrm{m} \text { scan } \\
(\mathrm{nm})\end{array}$ & $\begin{array}{c}\text { Total } \\
\text { insulator } \\
\text { thickness } \\
(\mathbf{n m})\end{array}$ \\
\hline $\begin{array}{l}\text { Bare native } \\
\text { oxide }\end{array}$ & 0.53 & 0.35 & 1.7 \\
\hline $\begin{array}{l}\text { Native oxide } \\
\text { with silicone oil }\end{array}$ & 0.339 & 0.334 & 1.6 \\
\hline o-dMeM & 0.37 & 0.35 & 1.9 \\
\hline oD-dMeM & 0.43 & 0.38 & 1.7 \\
\hline tDF-dMeM & 0.5 & 0.36 & 2.0 \\
\hline tDF-dMeC & 0.5 & 0.47 & 2.3 \\
\hline tDF-MedC & 0.45 & 0.47 & 2.9 \\
\hline hDF-tC & 1 & 0.6 & 3.5 \\
\hline tDF-tC & 0.56 & 0.53 & 2.8 \\
\hline nF-tC & 3.4 & 2.1 & 2.3 \\
\hline
\end{tabular}

Table II. RMS roughness (determined by AFM) and ellipsometry thickness values for the samples, measured immediately before breakdown testing. Thickness values were rounded to the nearest $0.1 \mathrm{~nm}$ owing to the precision of the measurement equipment; error in the thickness measurement was below $0.1 \mathrm{~nm}$ in all cases. The direct comparison between non-fluorinated hydrocarbon and perfluorinated carbon R-group SCAs are highlighted in blue. RMS roughness values are calculated from topographic images after median line matching, polynomial background subtraction, and excluding obvious scars with Gwyddion software. $25 \mu \mathrm{m} \times 25 \mu \mathrm{m}$ data are taken from the smoothest region in the $40 \mu \mathrm{m} \times 40 \mu \mathrm{m}$ original image (256 lines, $1 \mathrm{~Hz}, 0.3 \mathrm{~V}$ set point, contact mode with conductive diamond tip) and are included to illustrate the surface roughness without imaging artifacts or agglomerate bias.

E. Contact angle analysis: 
A Ramé-Hart model 500-F1 Advanced Goniometer with DropImage Advanced software was used to evaluate contact angles of DI water on each of the sample surfaces. A $5 \mu \mathrm{L}$ droplet and $2 \mu \mathrm{L}$ retraction were used to determine the advancing and receding contact angles, respectively.

\section{RESULTS AND DISCUSSION}

\section{A. I-V analysis}

Data analysis was conducted through I-V curve evaluation and Weibull failure analysis. With the aid of a MATLAB routine, the I-V data were matched through visual comparison to one of four characteristic curve types: 'Spike', 'Spike-Ohmic', 'Ohmic', and 'Discharge' (see Figure 2). Breakdown was defined as the maximum voltage withstood before sustained, significant current started flowing; 'Significant' current was taken to mean large current values relative to the low field leakage current (>1 nA), illustrating the need for I-V categorization. Automated detection of breakdown through monitoring high I-V slopes was not adequate due to false positives from noise and hardware sampling limits of the system. Breakdown strength was defined as the breakdown voltage divided by the total insulator thickness (oxide + SCA layer) determined by spectroscopic ellipsometry. The purpose of this method of evaluation is twofold: (1) to provide a practical and consistent definition of breakdown, and (2) to enhance the Weibull analysis by categorizing the breakdown types within a sample population. 

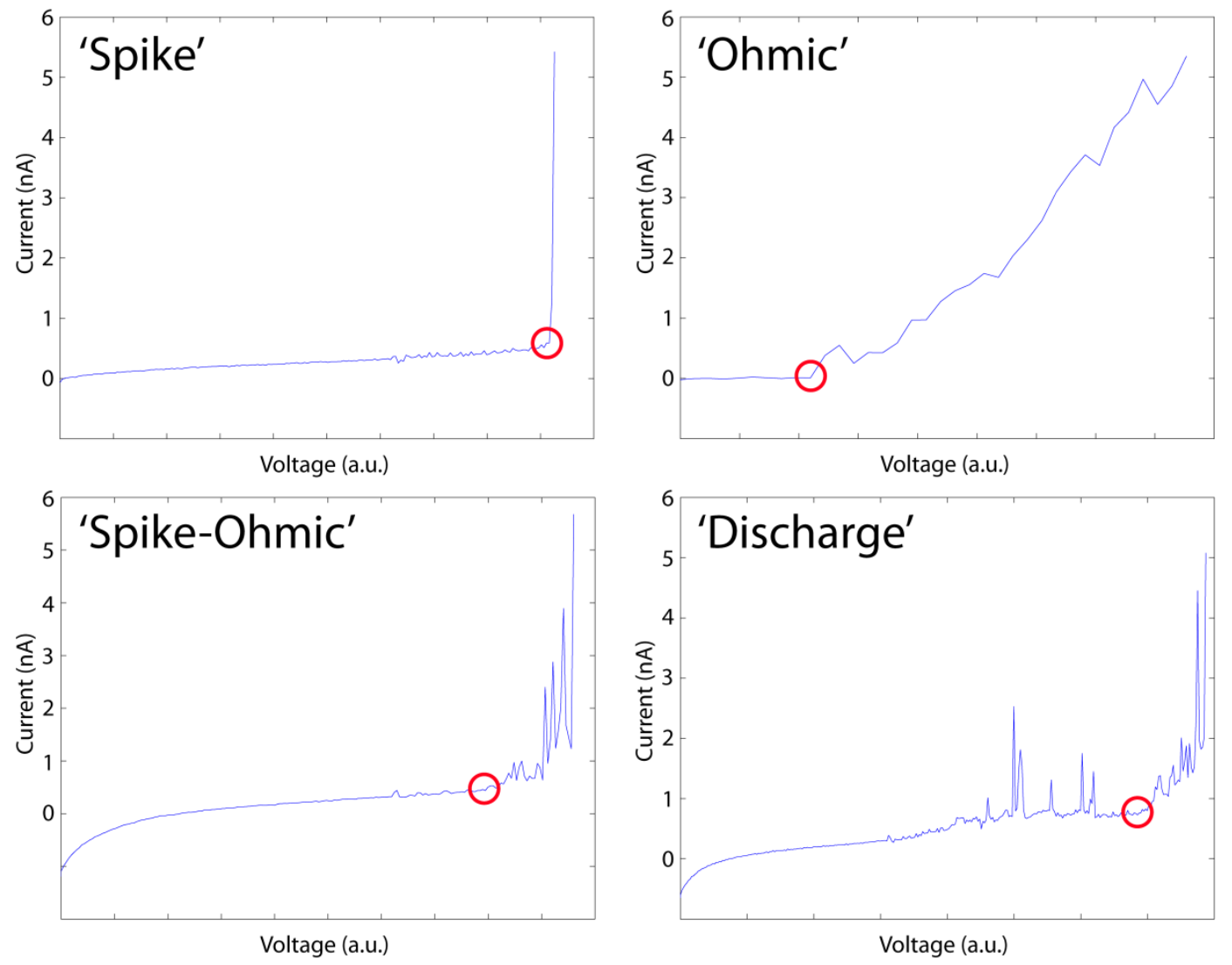

Figure 2. Characteristic I-V plots illustrating the four trends observed in the C-AFM tests. The highest voltage where 'significant' current flows, relative to the baseline leakage current, is defined as the breakdown voltage, denoted in each characteristic above by a red circle. 'Spike' is a sudden, abrupt increase in current. 'Ohmic' is a quasi-linear increase in current. 'Spike-Ohmic' is an increase in current with significant fluctuations superimposed over a linear trend. 'Discharge' involves current flows greater than $1 \mathrm{nA}$ which do not continue monotonically, but ultimately leads to a runaway increase in current. Each test was stopped when the recorded current reached $5 \mathrm{nA}$.

The definitions of breakdown are as follows, and are evaluated after the system has reached a current threshold of $5 \mathrm{nA}$ : 'Spike' breakdown is defined as a current increase of greater than $5 \mathrm{nA}$ over at most 3 data points, where the breakdown voltage is recorded at 
the data point with the highest voltage before such an increase. 'Ohmic' is defined as a quasi-linear increase in the I-V curve possessing a discontinuous slope (of greater than 5 data points) from the baseline current, with no more one instance of $50 \%$ variation in current between adjacent data points, whose breakdown voltage was identified at the onset of such a slope discontinuity. 'Discharge' is defined as having current flow greater than $1 \mathrm{nA}$ return to baseline levels for more than 3 data points, whose breakdown voltage is recorded at the highest voltage where the data exist on the baseline current. 'Spikeohmic', considered the remainder of the situations observed, is defined as an increase in current with greater than 1 instance of $50 \%$ variation in current between adjacent data points, after the onset of a discontinuity in slope observed over greater than 5 data points; breakdown voltage is recorded at the highest voltage where the data exist on the baseline before such a discontinuity.

\section{B. Weibull analysis}

To illustrate which breakdown type occurs, the Weibull plot data in Figure 3 have been color coded as follows. The inner marker color indicates the I-V type while the shape and outer marker color indicate the particular SCA molecule and sample treatment. The breakdown voltages are then divided by the thickness of the total insulator (SCA + oxide) to determine the breakdown strength, which are plotted according to a two-parameter Weibull distribution, and fit with a mixed two-parameter, two-mode maximum likelihood estimate, developed by Razali and Salih: ${ }^{32}$

$$
F(x)=w F_{1}(x)+(1-w) F_{2}(x)
$$


where $F_{1}(x)$ and $F_{2}(x)$ are separate two-parameter Weibull cumulative distribution functions, and $w$ is a weighting factor optimized by maximum likelihood estimation in MATLAB. Given the characteristic 'dogleg' shapes of the data, the model fits agree well, providing evidence that failure occurs by either one or the other of two different 'modes'.

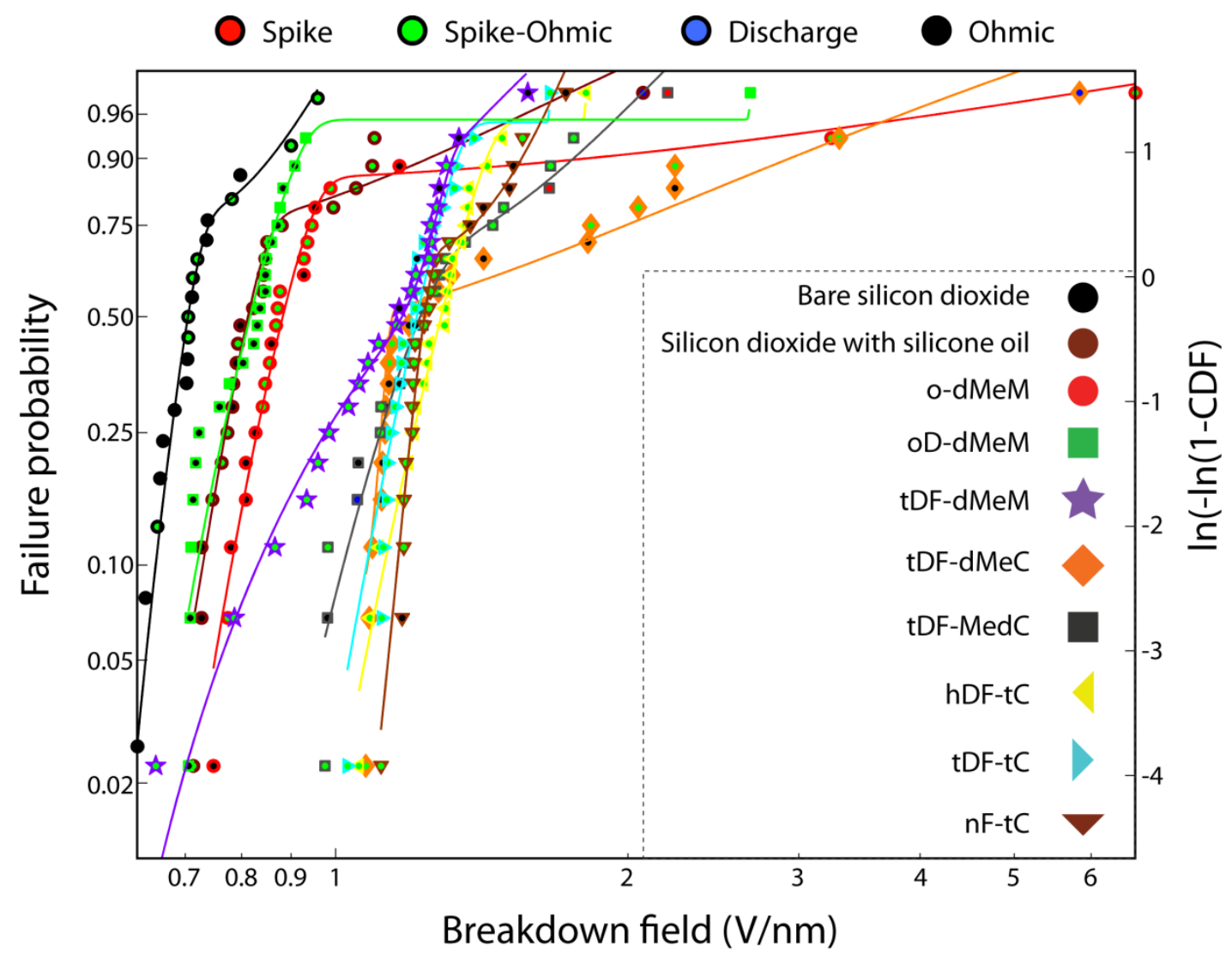

Figure 3. Weibull plot of C-AFM strike breakdown data for different SCA molecules bound to the indicated surfaces. The outer marker colors and symbol shapes correspond to samples according to the inset legend. Inner marker colors correspond to I-V type according to the legend above the graph. Two-parameter, two-mode Weibull maximum likelihood estimate fits to the data are included as the solid lines. Two equivalent 
ordinates are shown: the failure probability and the cumulative failure distribution function (CDF).

Several conclusions can be drawn from Figure 3. The bare native oxide is clearly the weakest insulator of the samples evaluated in this investigation. SCA attachment to the oxide increased the breakdown field in every case, with a maximum observed increase of $158 \%$ for $\mathrm{hDF}-\mathrm{tC}$, measured at $63.2 \%$ failure probability for the low field Weibull mode.

Failures of the bare native oxide are seen to occur largely with 'Ohmic' type I-V curves. As such, the 'Ohmic' breakdown process is likely dictated by quantum mechanical tunneling processes, which have been observed for thin oxides in several other studies. ${ }^{33-}$ 36 The native oxide with silicone oil, as well as the oxide with SCAs bearing saturated hydrocarbon R-groups, share similar distributions and exhibit significant 'Ohmic' failure. The silicone oil is expected to exist on the surface as a partial monolayer through adsorption to the silicon dioxide; further removal of the oil may be facilitated by exposure to SCA binding groups, although the mechanism is unclear. ${ }^{37}$ Regardless, like the native oxide, the residual oil is not the dominant resistance in the system; in that case no change in breakdown strength or distribution would be observed with SCA addition. In Figure 3, the separation along the abscissa between the silicone oil-treated and bare native oxide data indicates that residual silicone oil is likely bound to the surface. Strong hydrogen bonding between the silica surface silanols and silicone oil has been noted previously, but the silicone oil likely exists as a partial monolayer on the surface as the excess has been washed off in a good solvent. ${ }^{37}$ 'Spike' breakdown, because of its 
sudden, high slope, is estimated by the authors to possess greater electronic character than the other I-V types because of its abrupt nature relative to thermal processes; ${ }^{10,38}$ however, given the limited time resolution of our experiments, conclusive evidence of this character is not presented. The complex nature of the 'Spike-Ohmic' and 'Discharge' I-V types probably consist of both electronic and thermal breakdown mechanisms operating simultaneously, although 'Discharge' may also represent other pre-breakdown phenomena, such as those associated with avalanche quenching. ${ }^{10}$

The SCA hydrocarbon R-group is not seen to suppress tunneling behavior as evidenced by the presence of 'Ohmic' I-V data recorded from the octyldimethylmethoxysilane (o$\mathrm{dMeM})$ and n-octadecyldimethylmethoxysilane (oD-dMeM) samples. Other researchers have observed tunneling suppression with similar saturated hydrocarbon chains as an effect of increasing the tunneling barrier height. ${ }^{2}$ The SAMs in this investigation are intentionally disordered through formation of a partial monolayer to ensure that a particular SCA's propensity to form defects, from steric or other differences, did not influence the breakdown results. This precaution was taken to further isolate SCA chemistry as the only independent variable in the experiment. Water contact angle data, listed in Table III, show that both hydrocarbon and fluorocarbon SCAs exhibit hydrophobicity well below what is expected for densely packed SAMs indicating molecular disorder; fully dense monolayers are expected to exhibit advancing contact angles of approximately $115^{\circ}$ and $112^{\circ}$ for fluorinated and hydrocarbon functionality, respectively. ${ }^{39-40}$ It is useful to note that the electrical resistance of the SCA layer has 
been observed to be significantly higher than that of the native oxide, ${ }^{2}$ as illustrated in Figure 3 .

\begin{tabular}{|l|c|c|c|}
\hline \multicolumn{1}{|c|}{ SCA } & $\boldsymbol{\theta}_{\text {adv }}$ [deg] & $\boldsymbol{\theta}_{\text {rec }}[\mathbf{d e g}]$ & $\boldsymbol{\theta}_{\text {hys }}$ [deg] \\
\hline o-dMeM & $83.8 \pm(1.2)$ & $62.5 \pm(1.2)$ & 21.3 \\
\hline $\begin{array}{l}\text { oD- } \\
\text { dMeM }\end{array}$ & $83.7 \pm(0.4)$ & $63.5 \pm(1.7)$ & 20.2 \\
\hline $\begin{array}{l}\text { tDF- } \\
\text { dMeM }\end{array}$ & $86.3 \pm(0.7)$ & $66.0 \pm(2.6)$ & 20.3 \\
\hline $\begin{array}{l}\text { tDF- } \\
\text { dMeC }\end{array}$ & $86.6 \pm(0.2)$ & $66.9 \pm(1.3)$ & 19.7 \\
\hline $\begin{array}{l}\text { tDF- } \\
\text { MedC }\end{array}$ & $88.3 \pm(0.6)$ & $66.6 \pm(4.0)$ & 20.4 \\
\hline hDF-tC & $90.4 \pm(0.1)$ & $69.8 \pm(2.5)$ & 20.6 \\
\hline tDF-tC & $88.3 \pm(2.8)$ & $74.1 \pm(2.1)$ & 14.2 \\
\hline nF-tC & $88.9 \pm(0.0)$ & $69.4 \pm(3.3)$ & 19.5 \\
\hline
\end{tabular}

Table III. Water contact angle analysis for various SCA-SAM samples. The low advancing contact angles and high hysteresis values, relative to what is expected for a fully dense SCA monolayer, imply that the SCA treatments created partial monolayers with significant molecular disorder. $\theta_{\mathrm{adv}}$ and $\theta_{\text {rec}}$, indicate average advancing and receding water contact angles \pm one standard deviation, respectively; $\theta_{\text {hys }}$ indicates the average contact angle hysteresis.

At high breakdown fields, the number of SCA binding groups leads to subtle effects seen in Figure 3. For fluorinated SCAs with eight carbons, the slope is large at high breakdown fields for SCAs with trifunctional binding groups, and monotonically decreases as the number of binding groups is reduced, indicating higher consistency with more binding groups. However, the breakdown strength of these high field tails decreases in absolute terms when reducing the number of binding groups. Therefore, 
fewer binding groups yield potentially higher breakdown strengths at the cost of lower consistency. This trend can be understood as a consequence of local SCA packing: molecules with fewer binding groups experience less steric hindrance, allowing the possibility of higher molecular packing efficiency in the plane of the substrate, and thus greater breakdown strength. However, the high molecular packing efficiency is not consistent because of the reduced probability of surface attachment associated with fewer binding groups. In contrast, SCA molecules with multiple binding groups suffer from steric packing problems but adhere more consistently to the surface, yielding a more reliable electrically-insulating SAM on average. Previously observed ${ }^{41}$ high field Weibull 'tails' have been ignored largely ignored as non-idealities.

Fluorinated SCA functionalities are thought to suppress tunneling and increase breakdown strength through high field electron attachment behavior observed in both sulfur hexafluoride ${ }^{42}$ and perfluorinated liquids. ${ }^{43}$ Additionally, the highly electronegative nature of fluorine and the ionic C-F bond may cause scattering of ballistic electrons ${ }^{44-45}$ and act as electronic traps. ${ }^{10}$ Additionally, molecular dipole moments have been correlated directly with mean electron scattering cross-sections. ${ }^{46}$ Although it is not presently clear which mechanism is primarily responsible for breakdown suppression in fluorinated compounds, their comparison with hydrogenated analogs illustrates the effect of bonding character differences on dielectric breakdown. It can be seen in Figure 3 that SCAs with fluorinated R- outperformed those which were not. The number of 'Ohmic' events also decreased greatly with fluorination, which were largely replaced with 'SpikeOhmic' events. The greater thickness of the fluorinated SCA SAMs relative to their 
hydrocarbon counterparts contributes to tunneling suppression and increased breakdown strength because of exponential tunneling current decay with distance. However, a direct comparison between o-dMeM and its fluorinated analog tDF-dMeM reveals that fluorination directly affects the SAM's ability to resist electrical breakdown. Comparing the breakdown strength values at the $63.2 \%$ failure probability, the fluorinated SCA exhibits a $48 \%$ increase relative to its hydrocarbon counterpart. In a closer comparison of the data in Figure 3, the only difference between the two samples is R-group fluorination: the molecules possess the same hydrocarbon backbone length, similar RMS roughness values $(0.01 \mathrm{~nm}, 25 \mu \mathrm{m} \times 25 \mu \mathrm{m}$ scans, Table II), and nearly identical SAM thicknesses ( $1 \AA$ different).

Although the scope of this research does not permit detailed analysis of the breakdown mechanisms of fluorinated SCAs, we hypothesize that thermal processes are involved in conduction, either through field-assisted or defect-mediated hopping mechanisms because of the large proportion of observed 'Spike-Ohmic' I-V events. ${ }^{48-49}$ However, it is believed that this thermally influenced conduction competes with electron scattering or capture by highly polar C-F bonds ${ }^{10}$ or ionized fluorine atoms,${ }^{50}$ leading to tunneling suppression. As described above, it is assumed that tunneling is responsible for the majority of conduction for disordered hydrocarbon SCAs, native oxide, and residual silicone oil on native oxide samples.

The internal electric field experienced by the SCA per bond is expected to be similar between the molecules examined, regardless of their length, due to the lack of defects in 
such short chains. This is consistent with the data in Figure 3, in which the homologous series of fluorinated SCAs is seen to exhibit similar breakdown strengths largely independent of chain length, an effect which has been observed previously. ${ }^{22-23,51}$ To further illustrate the chemical effects of fluorination on breakdown, the data in Figure 3 were normalized by the dielectric constant of the particular SCA molecule, as seen in Figure $4 .^{28}$ This represents the internal electric field experienced by an average molecule in the SAM, allowing further normalization of physical differences between the SCA chemistries. As the dielectric constants of the SCAs in this investigation are all within $16 \%$, it is expected that the Weibull plots in Figures 3 and 4 should also be similar. Indeed, only the relative position of the data from the silicone oil on silicon dioxide changes significantly relative to the other data sets, as the silicone oil layer is extremely thin. As such, fluorinated SCAs can be seen to withstand higher breakdown strengths than their hydrocarbon counterparts as evidenced by their higher internal electric fields at breakdown. 


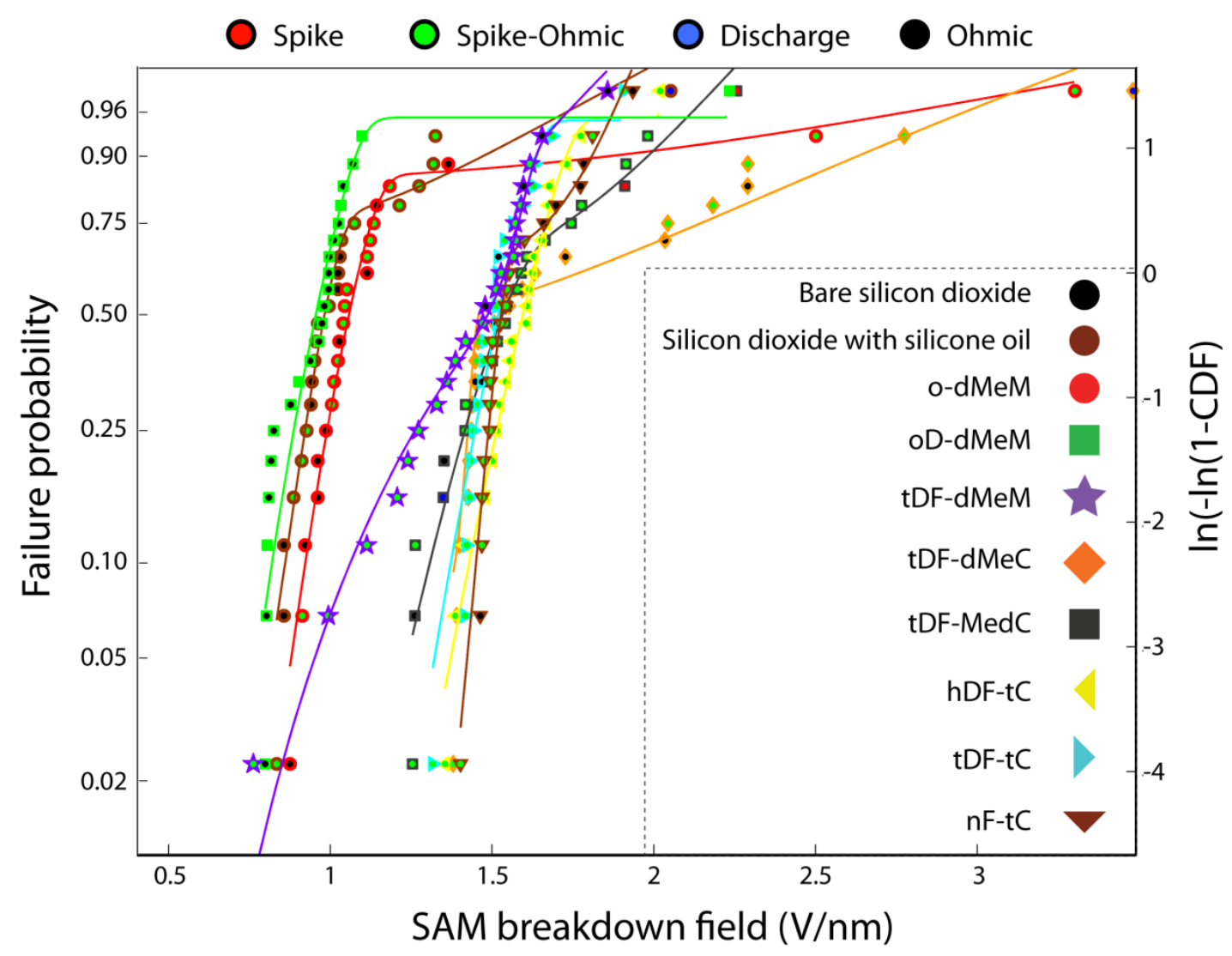

Figure 4. Weibull plot of the breakdown data normalized by the dielectric constant of the SCA molecules. The total insulator thicknesses shown in Table II include the $1.5 \mathrm{~nm}$ native oxide, yielding the SAM thickness used to calculate the internal breakdown field. DC dielectric constants were taken as the square of the bulk refractive index of the SCA. ${ }^{28}$ The two-parameter, two-mode Weibull maximum likelihood estimate fits of the data are included as solid lines.

\section{CONCLUSIONS}

It has been demonstrated that vapor-deposited SCA monolayers on native oxide can dramatically improve nanoscale dielectric breakdown strength, in some cases by up to 158\%. Through the formation of deliberately disordered partial monolayers, direct comparison between saturated hydrocarbon and fluorocarbon moieties was conducted 
using C-AFM. It was observed that fluorination increases the dielectric breakdown strength and helps suppress tunneling. The SCA length did not affect the breakdown strength significantly, and high field breakdown was seen to correlate with the number of SCA linking groups. Normalization of the breakdown strength by the dielectric constant of the individual SCA molecules yielded similar trends to the raw Weibull data. Electron attachment, capture, or scattering effects from the highly electronegative fluorine atom are thought to be responsible for the increase in breakdown strength achieved by fluorination.

\section{ACKNOWLEDGMENTS}

The authors thank Louis Perez at UCSB and Daniel Smaltz at Harvard for assistance with the SCA modification, the Whitesides group at Harvard for the use of the contact angle apparatus, and Professors Edward J. Kramer and Craig J. Hawker at UCSB for their many helpful suggestions. This work was performed in part at Harvard University's Center for Nanoscale Systems, a member of the National Nanotechnology Infrastructure Network, which is supported by the National Science Foundation under NSF award no. ECS-0335765 and supported primarily by the MRSEC program of the National Science Foundation under award no. DMR-0820484. 


\section{REFERENCES}

1. DiBenedetto, S. A.; Facchetti, A.; Ratner, M. A.; Marks, T. J., Molecular SelfAssembled Monolayers and Multilayers for Organic and Unconventional Inorganic Thin-Film Transistor Applications. Adv. Mat. 2009, 21 (14-15), 14071433.

2. Boulas, C.; Davidovits, J. V.; Rondelez, F.; Vuillaume, D., Suppression of Charge Carrier Tunneling through Organic Self-Assembled Monolayers. Phys. Rev. Lett. 1996, $76(25)$, 4797-4800.

3. Jedaa, A.; Burkhardt, M.; Zschieschang, U.; Klauk, H.; Habich, D.; Schmid, G.; Halik, M., The Impact of Self-Assembled Monolayer Thickness in Hybrid Gate Dielectrics for Organic Thin-Film Transistors. Org. Electr. 2009, 10 (8), 14421447.

4. Ha, Y. G.; Emery, J. D.; Bedzyk, M. J.; Usta, H.; Facchetti, A.; Marks, T. J., Solution-Deposited Organic-Inorganic Hybrid Multilayer Gate Dielectrics. Design, Synthesis, Microstructures, and Electrical Properties with Thin-Film Transistors. J. Am. Chem. Soc. 2011, 133 (26), 10239-50.

5. Yoon, M. H.; Facchetti, A.; Marks, T. J., $\sigma-\pi$ Molecular Dielectric Multilayers for Low-Voltage Organic Thin-Film Transistors. Proc. Natl. Acad. Sci. U S A 2005, 102 (13), 4678-82.

6. Aswal, D. K.; Lenfant, S.; Guerin, D.; Yakhmi, J. V.; Vuillaume, D., Self Assembled Monolayers on Silicon for Molecular Electronics. Analytica Chimica Acta 2006, 568 (1-2), 84-108. 
7. Aswal, D. K.; Petit, C.; Salace, G.; Guérin, D.; Lenfant, S.; Yakhmi, J. V.; Vuillaume, D., Role of Interfaces on the Direct Tunneling and the Inelastic Tunneling Behaviors through Metal/Alkylsilane/Silicon Junctions. phys. stat. solid. (a) 2006, 203 (6), 1464-1469.

8. DiBenedetto, S. A.; Facchetti, A.; Ratner, M. A.; Marks, T. J., Charge Conduction and Breakdown Mechanisms in Self-Assembled Nanodielectrics. J. Am. Chem. Soc. 2009, 131 (20), 7158-7168.

9. Diebold, R. M.; Clarke, D. R., Smooth, Aggregate-Free Self-Assembled Monolayer Deposition of Silane Coupling Agents on Silicon Dioxide. Langmuir 2012, 28 (44), 15513-15520.

10. Dissado, L. A.; Fothergill, J. C., Electrical Degradation and Breakdown in Polymers. Vol. 9; Peter Peregrinus: London, 1992.

11. Guo, N.; DiBenedetto, S. A.; Tewari, P.; Lanagan, M. T.; Ratner, M. A.; Marks, T. J., Nanoparticle, Size, Shape, and Interfacial Effects on Leakage Current Density, Permittivity, and Breakdown Strength of Metal Oxide-Polyolefin Nanocomposites: Experiment and Theory. Chem. Mat. 2010, 22 (4), 1567-1578.

12. Li, J. Y.; Zhang, L.; Ducharme, S., Electric Energy Density of Dielectric Nanocomposites. Appl. Phys. Lett. 2007, 90 (13), 132901.

13. Sire, C.; Blonkowski, S.; Gordon, M. J.; Baron, T., Statistics of Electrical Breakdown Field in $\mathrm{HfO}_{2}$ and $\mathrm{SiO}_{2}$ Films from Millimeter to Nanometer Length Scales. Appl. Phys. Lett. 2007, 91 (24), 242905. 
14. Teichert, C.; Beinik, I., Conductive Atomic-Force Microscopy Investigation of Nanostructures in Microelectronics. In Scanning Probe Microscopy in Nanoscience and Nanotechnology 2, Springer: 2011; pp 691-721.

15. Murrell, M. P.; Welland, M. E.; O’Shea, S. J.; Wong, T. M. H.; Barnes, J. R.; McKinnon, A. W.; Heyns, M.; Verhaverbeke, S., Spatially Resolved Electrical Measurements of $\mathrm{SiO}_{2}$ Gate Oxides using Atomic Force Microscopy. Appl. Phys. Lett. 1993, 62 (7), 786-788.

16. Iglesias, V.; Porti, M.; Nafría, M.; Aymerich, X.; Dudek, P.; Bersuker, G., Dielectric Breakdown in Polycrystalline Hafnium Oxide Gate Dielectrics Investigated by Conductive Atomic Force Microscopy. J. Vac. Sci. Tech. B 2011, $29(1), 01 \mathrm{AB} 02$.

17. Polspoel, W.; Vandervorst, W.; Aguilera, L.; Porti, M.; Nafria, M.; Aymerich, X., Nanometer-Scale Leakage Measurements in High Vacuum on de-Processed Highk Capacitors. Microelectron. Reliab. 2008, 48 (8-9), 1521-1524.

18. Porti, M.; Nafría, M.; Aymerich, X.; Olbrich, A.; Ebersberger, B., NanometerScale Electrical Characterization of Stressed Ultrathin $\mathrm{SiO}_{2}$ Films Using Conducting Atomic Force Microscopy. Appl. Phys. Lett. 2001, 78 (26), 4181.

19. Zhang, L.; Mitani, Y., Structural and Electrical Evolution of Gate Dielectric Breakdown Observed by Conductive Atomic Force Microscopy. Appl. Phys. Lett. 2006, 88 (3), 032906.

20. Kremmer, S.; Teichert, C.; Pischler, E.; Gold, H.; Kuchar, F.; Schatzmayr, M., Characterization of Silicon Gate Oxides by Conducting Atomic Force Microscopy. Surf. Interface. Anal. 2002, 33 (2), 168-172. 
21. Hourani, W.; Gautier, B.; Militaru, L.; Albertini, D.; Descamps-Mandine, A.; Arinero, R., Influence of the Surrounding Ambient on the Reliability of the Electrical Characterization of Thin Oxide Layers using an Atomic Force Microscope. Microelectron. Reliab. 2011, 51 (12), 2097-2101.

22. Wold, D. J.; Frisbie, C. D., Fabrication and Characterization of Metal-MoleculeMetal Junctions by Conducting Probe Atomic Force Microscopy. J. Am. Chem. Soc. 2001, 123 (23), 5549-5556.

23. Zhao, J.; Uosaki, K., Dielectric Properties of Organic Monolayers Directly Bonded on Silicon Probed by Current Sensing Atomic Force Microscope. Appl. Phys. Lett. 2003, 83 (10), 2034.

24. Lee, T.; Wang, W.; Klemic, J. F.; Zhang, J. J.; Su, J.; Reed, M. A., Comparison of Electronic Transport Characterization Methods for Alkanethiol Self-Assembled Monolayers. J. Phys. Chem. B 2004, 108 (25), 8742-8750.

25. Park, J. Y.; Qi, Y. B.; Ashby, P. D.; Hendriksen, B. L. M.; Salmeron, M., Electrical Transport and Mechanical Properties of Alkylsilane Self-Assembled Monolayers on Silicon Surfaces Probed by Atomic Force Microscopy. J. Chem. Phys. 2009, 130 (11).

26. Kuikka, M. A.; Li, W.; Kavanagh, K. L.; Yu, H. Z., Nanoscale Electrical and Structural Characterization of Gold/Alkyl Monolayer/Silicon Diode Junctions. $J$. Phys. Chem. C 2008, 112 (24), 9081-9088.

27. Haag, R.; Rampi, M. A.; Holmlin, R. E.; Whitesides, G. M., Electrical Breakdown of Aliphatic and Aromatic Self-Assembled Monolayers used as Nanometer-Thick Organic Dielectrics. J. Am. Chem. Soc. 1999, 121 (34), 7895-7906. 
28. See supplemental information.

29. Kotzsch, H. J.; Vahlensieck, H. J., US Pat. No. 3985781.

30. Herzinger, C.; Johs, B.; McGahan, W.; Woollam, J.; Paulson, W., Ellipsometric Determination of Optical Constants for Silicon and Thermally Grown Silicon Dioxide via a Multi-Sample, Multi-Wavelength, Multi-Angle Investigation. $J$. Appl. Phys. 1998, 83 (6), 3323-3336.

31. Fuierer, R. Basic Operation Procedures for the Asylum Research MFP-3D Atomic Force Microscope. Whitepaper. Asylum Research: 2006.

32. Razali, A. M.; Salih, A. A., Combining Two Weibull Distributions using a ixing Parameter. Eur. J. Sci. Res. 2009, 31 (2), 296-305.

33. Ghetti, A.; Liu, C. T.; Mastrapasqua, M.; Sangiorgi, E., Characterization of Tunneling Current in Ultra-Thin Gate Oxide. Solid-State Electron. 2000, 44, $1523-1531$.

34. Ravindra, N. M.; Zhao, J., Fowler-Nordheim Tunneling in Thin $\mathrm{SiO}_{2}$ Films. Smart Mater. Struct. 1992, 1 (3), 197.

35. Fisher, J. C.; Giaever, I., Tunneling Through Thin Insulating Layers. J. Appl. Phys. 1961, 32 (2), 172-177.

36. Maserjian, J.; Zamani, N., Behavior of the $\mathrm{Si} / \mathrm{SiO}_{2}$ Interface observed by FowlerNordheim Tunneling. J. Appl. Phys. 1982, 53 (1).

37. Papirer, E., In Adsorption on Silica Surfaces, Marcek Dekker: New York, 2000; Chapter 19, pgs 622-638. 
38. Fothergill, J. C. In Ageing, Space Charge and Nanodielectrics: Ten Things we don't know about Dielectrics, Solid Dielectrics, 2007. ICSD'07. IEEE International Conference on, IEEE: 2007; pp 1-10.

39. Schift, H.; Saxer, S.; Park, S.; Padeste, C.; Pieles, U.; Gobrecht, J., Controlled CoEvaporation of Silanes for Nanoimprint Stamps. Nanotechnology 2005, 16 (5), S171-S175.

40. Srinivasan, U.; Houston, M. R.; Rowe, R. T.; Maboudian, R. In Self-Assembled Fluorocarbon Films for Enhanced Stiction Reduction, International Conference on Solid State Sensors and Actuators, Chicago, 16-19 Jun 1997; Chicago, 1997; pp 1399-1402.

41. Schlitz, R. A.; Yoon, K.; Fredin, L. A.; Ha, Y.; Ratner, M. A.; Marks, T. J.; Lauhon, L. J., Weibull Analysis of Dielectric Breakdown in a Self-Assembled Nanodielectric for Organic Transistors. J. Phys. Chem. Lett. 2010, 1 (22), 32923297.

42. Baird, J. K., Kinetics of Electron Capture by $\mathrm{SF}_{6}$ in Solution. Can. J. Chem. 1977, 55(11): 2133-2143.

43. Spyrou, S. M.; Sauers, I.; Christophorou, L. G., Electron Attachment to the Perfluoroalkanes $n-\mathrm{C} N \mathrm{~F}_{2} N_{+2}(N=1-6)$ and $i-\mathrm{C}_{4} \mathrm{~F}_{10}$. J. Chem. Phys. 1983, 78 (12), $7200-7216$.

44. Chesnyi, A. S.; Rambidi, N. G., Elastic Scattering of fast Electrons by Highly Polar Molecules. J. Chem. Phys. 1977, 26, 155-162.

45. Christophorou, L. G.; James, D. R.; Mathis, R. A., Dielectric Gas Mixtures with Polar Components. J. Phys. D: Appl. Phys 1981, 14, 675-692. 
46. Christophorou, L. G.; Christodoulides, A. A., Scattering of Thermal Electrons by Polar Molecules. J. Phys. B: Atom. Molec. Phys. 1969, 2 (1), 71.

47. Beran, J. A.; Kevan, L., Molecular Electron Ionization Cross Sections at 70 eV. J. Phys. Chem. 1969, 73 (11), 3866-3876.

48. Holmlin, R. E.; Haag, R.; Chabinyc, M. L.; Ismagilov, R. F.; Cohen, A. E.; Terfort, A.; Rampi, M. A.; Whitesides, G. M., Electron Transport through Thin Organic Films in Metal-Insulator-Metal Junctions based on Self-Assembled Monolayers. J. Am. Chem. Soc. 2001, 123 (21), 5075-5085.

49. Paracchini, C.; Dallacasa, V.; Romano, L., The Role of the Internal Field on the Electronic Transport in Insulators. Electrical Insulation, IEEE Transactions on 1991, $26(2), 222-227$.

50. Christophorou, L.; Mathis, R.; James, D.; McCorkle, D., On the Role of Electron Attachment in the Breakdown Strength of Gaseous Dielectrics. J. Phys. D: Appl. Phys. 1981, 14 (10), 1889.

51. Mbindyo, J. K.; Mallouk, T. E.; Mattzela, J. B.; Kratochvilova, I.; Razavi, B.; Jackson, T. N.; Mayer, T. S., Template Synthesis of Metal Nanowires Containing Monolayer Molecular Junctions. J. Am. Chem. Soc. 2002, 124 (15), 4020-4026. 